\title{
Peripheral neuropathy associated with the sicca syndrome
}

\author{
RP KENNETT, AE HARDING \\ From the National Hospital for Nervous Diseases, and Hammersmith Hospital, London, UK
}

SUMMARY Three patients with the sicca syndrome and chronic sensory neuropathy are described; in two of them neuropathy was the presenting feature of the disease. The sicca syndrome can give rise to a characteristic neurological syndrome comprising areflexia and asymmetrical sensory loss, particularly of proprioception, in the limbs. This is often associated with tonic pupils and trigeminal anaesthesia.

Sjögren's syndrome consists of keratoconjunctivitis sicca, xerostomia and a connective tissue disease, usually rheumatoid arthritis. The term sicca syndrome is used to describe the first two features occurring without an associated connective tissue disease. Each has been described with a wide range of other manifestations including lymphadenopathy, lymphoma, pseudolymphoma, splenomegaly, leucopenia, purpura, vasculitis, renal tubular defects, and peripheral neuropathy. Immunological abnormalities with hypergammaglobulinaemia and the presence of extractable nuclear antigens and multiple autoantibodies occur in both syndromes. ${ }^{1}$

In previous reports of peripheral neuropathy associated with connective tissue diseases, the distinction between Sjögren's and sicca syndromes has rarely been made. In Sjögren's syndrome peripheral neuropathy is generally thought to be rare. ${ }^{2}$ It occurred in 10 of 109 patients reviewed by Kaltreider and Talal, although two of these had sicca syndrome alone. ${ }^{3}$ This paper describes three patients who had longstanding sensory neuropathy associated with sicca syndrome; in two of them neuropathy was the presenting feature of the disease.

Address for reprint requests: Dr R Kennett, Department of Clinical Neurology, Institute of Neurology, Queen Square, London, WC1N 3BG, UK.

Received 12 March 1985. Accepted 10 May 1985

\section{Case reports}

Case 1

This female, now aged 63 years, was first aware of unsteadiness of gait in 1962, although this had been apparent to relatives before that time. The disability became progressively more severe, but she was able to continue workingo until 1973, using a stick for support. She then noticed impairment of sensation in the hands, being unsure of tex- -0 tures and unable to identify objects without visual clues. For four years she had been aware of difficulty locating hero left arm in space, especially in the dark. Temperature per? ception was normal. She had been unable to write for eighteen years because of difficulty in controlling a pen and she found that her hands "jumped". She could not do up buttons or shoe laces but was able to feed and dress herself. She denied weakness but the left foot tended to slap on the ground and her arms were poorly co-ordinated, especially the left. At the time of examination she had been chairbound for one year.

Her eyes had been dry for at least 16 years, and she had impaired saliva production which did not cause difficulty with eating. A dental clearance was performed in 1965 because of frequent gum infections. She had suffered from episodic diarrhoea for fourteen years, at worst passing motions four times a day. When examined in 1985, she was thin but did not appear unwell. Both eyes were dry but the tongue was moist. The pupils were $4 \mathrm{~mm}$ in diameter and there was reduced constriction in response to light, particularly on the left. The accommodation reaction was relatively preserved, and symmetrical. There was marked bilateral constriction in response to $0.1 \%$ pilocarpine. Pain and touch appreciation was impaired in the distribution of the left trigeminal nerve and of the first division on the right. Both corneal responses were reduced, especially on the right. There was wasting of the intrinsic muscles of both 
hands and feet, and the ulnar side of the left forearm. There was bilateral weakness (MRC grade $4+$ ) of wrist and finger flexion, finger extension and the small hand muscles. Power was normal in the legs. All the tendon reflexes were absent. There was mild pseudo-athetosis of the outstretched hands and moderately severe limb ataxia which was accentuated by eye closure. Light touch appreciation was absent below the left clavicle, right elbow, and the mid thighs, and pain was not felt distal to the knees and elbows. Proprioception was lost at the fingers, wrists and elbows, and impaired in the shoulders, knees and ankles. Large movements of the toes were just detected. Vibration sense was absent below the shoulders, right knee and left iliac crest. The two-point discrimination threshold was more than $7 \mathrm{~cm}$ on the index fingers.

Abnormal investigations included a weakly positive Latex rheumatoid factor, positive antinuclear antibody (1/160 speckled pattern) and extractable nuclear antigen (1/2 SS-B), and hypergammaglobulinaemia on serum protein electrophoresis. Schirmer's test was positive and lip biopsy showed mononuclear cell infiltration with no detectable salivary glands in a large specimen. Sensory nerve action potentials were undetectable on recording from the median, ulnar and sural nerves. Motor nerve conduction velocities in the median, ulnar and peroneal nerves were normal at 52,54 and $40 \mathrm{~m} / \mathrm{s}$ respectively. Tests of autonomic function showed increased carotid sinus sensitivity, postural hypotension with preserved pressor response to stress and an abnormal response to the Valsalva manoeuvre, indicating an afferent lesion with retained efferent function. The histamine flare response was abnormal distally in the limbs.

Treatment with corticosteroids in $\mathbf{1 9 8 0}$ did not alter the course of her neurological illness.

\section{Case 2}

This female was aged $70 \mathrm{yr}$ when last reviewed in 1970 . Sjögren's syndrome was diagnosed in 1958 when she developed dental caries, parotid swelling and xerostomia. A salivary gland biopsy showed intense lymphocytic infiltration. A Schirmer's test was positive in 1967, when there was also Rose Bengal staining of the left bulbar conjunctiva. There were no symptoms of xerophthalmia.

In June 1966 she noticed pain and numbness in the little finger of the right hand. An ulnar nerve transposition failed to alleviate her symptoms, and soon afterwards she developed tingling and "electric" sensations in all the fingers of the right hand. Three months later, similar symptoms appeared in the left hand. The hands felt "wooden", "heavy" and "dead", were clumsy and she was unable to dress or feed herself, although there was no weakness. When seen in 1970 she gave an 18 month history of progressive difficulty in walking. She required a wall for support and her unsteadiness was worse in the dark. The right foot had been numb over that time but she felt that the left was normal and denied weakness.

On examination, the cranial nerves were normal. There was slight wasting of the intrinsic muscles of both hands with mild weakness. Muscle bulk was normal in the legs, but dorsiflexion and eversion of the ankles were weak bilaterally. All the tendon reflexes were absent but the abdominal and plantar responses were normal. There was bilateral hypalgesia and hypaesthesia below the wrists and ankles. Vibration was only perceived over the shoulders and sternum. Position sense was absent in the fingers and toes and grossly impaired in all other limb joints, especially in the arms. There was marked sensory ataxia and pseudo-athetosis of the upper limbs and gait ataxia with Rombergism.

Routine haematological and biochemical tests, including glucose tolerance, serum protein electrophoresis and vitamin B12, were normal. Porphyrins were not detected in the urine. No sensory nerve action potentials were detectable recording from the median and ulnar nerves. Median, ulnar and peroneal motor nerve conduction velocities were normal at 54,56 and $46 \mathrm{~m} / \mathrm{s}$ respectively.

There was little objective change on examination over two years of review before the patient died in October 1970. Prednisolone, $15 \mathrm{mg}$ a day reducing over 3 months, did not affect the progress of her illness. A post mortem examination was not performed.

\section{Case 3}

This 33-year-old female has been described briefly elsewhere. 4 She developed numbness and clumsiness of the right hand in 1973. This remained static for three years before she noticed loss of sensation involving her right foot. In 1980 she rapidly became aware of numbness of the left hand associated with a feeling of constriction around the wrist. For two years prior to assessment she had noticed progressive numbness of her face and scalp, and mild unsteadiness of gait. On direct questioning she admitted to some dryness of the eyes for a few years, and that her hands were blue and painful if exposed to cold.

On examination in 1983 she was extremely thin (weight $30 \mathrm{~kg}$ ). The pupils were irregular, asymmetrical and reacted poorly to light but relatively well to accommodation. There was hypersensitivity to $0.1 \%$ pilocarpine. Pain and touch appreciation was reduced in the distribution of both trigeminal nerves, with reduced corneal reflexes. There was no muscle wasting or weakness, but all the tendon reflexes were absent except for the left biceps jerk. There was hypalgesia and hypaesthesia distal to the mid forearms and knees. Vibration sense was absent below the left clavicle and right elbow, and reduced below the knees. Joint position sense was absent in the fingers and toes, and impaired at both wrists and at the left elbow. The two point discrimination threshold was greater than $5 \mathrm{~cm}$ on the index fingers. The gait was ataxic with Rombergism.

Schirmer's test was positive and there was lymphocytic infiltration of a minor salivary gland. Other abnormal investigations included a positive rheumatoid Latex test, raised antinuclear antibodies (1/320) and extractable nuclear antigen, and elevated levels of immunoglobulin $M$ in the serum. Sensory nerve action potentials were undetectable on recording from the median, ulnar and sural nerves. Motor nerve conduction velocity in the median, ulnar and peroneal nerves was normal at 55,51 and $45 \cdot 5 \mathrm{~m} / \mathrm{s}$.

\section{Discussion}

The three patients reported all suffered from a chronic peripheral sensory neuropathy with striking 
impairment of position sense, particularly in the upper limbs, indicating involvement predominantly of large myelinated fibres. ${ }^{5}$ This was the most disabling feature of their illness. Sensory symptoms often started in one limb but were not confined to the territory of a single peripheral nerve. The sensory deficit tended to become symmetrical with time. Although symptoms had been present for eighteen and ten years respectively in cases 1 and 3, motor involvement remained inconspicuous. Sensory impairment in the trigeminal nerve territory was seen in two patients; again there was a tendency for this to be asymmetrical. These two patients also had tonic pupils, with pharmacological evidence of parasympathetic denervation. Slow progression of the neuropathy over many years was another characteristic feature.

A clear distinction between Sjögren's and sicca syndrome has not been made in previous reports of patients with associated peripheral neuropathy. ${ }^{367}$ Kaltreider and $\mathrm{Talal}^{3}$ reported two cases of sicca syndrome and sensorimotor neuropathy. Trigeminal involvement occurred in one. Peripheral neuropathy in the other patients in their series may have been related to the associated connective tissue disease: the clinical features in some were similar to those seen in rheumatoid arthritis. ${ }^{8}$

Although Weber' referred to a patient with Sjögren's and Adie's syndromes, and Attwood and Poser ${ }^{10}$ described a patient with anisocoria and impaired pupillary reactions, detailed assessment of pupillary responses has not been described in cases of Sjögren's syndrome and neuropathy. The occurrence of tonic pupils exhibiting light-near dissociation, due to parasympathetic denervation of the pupillary sphincter, has been documented in a number of peripheral neuropathies, particularly in diabetes. ${ }^{11}$

Nerve biopsies performed in patients with peripheral neuropathy and Sjögren's syndrome have occasionally shown evidence of medium to small vessel vasculitis. ${ }^{3}$ Peripheral nerve histology has not been described in patients similar to those reported here, and their clinical features were not typical of those seen in vasculitic neuropathy. ${ }^{12}$

In two of our three patients the clinical features of sicca syndrome was subtle and the diagnosis was not made until symptoms of neuropathy had been pres- ent for over ten years. This diagnosis should be considered in cases of chronic sensory axonal neuropathy, particularly if proprioceptive loss is prominent and there are pupillary abnormalities and trigeminal involvement. Investigations should include auto-antibody studies and salivary gland biopsy.

We thank Drs CJ Earl, R Capildeo, GRV Hughes, and Professor RW Gilliatt for permission to report patients under their care.

\section{References}

${ }^{1}$ Hughes GRV. Connective Tissue Diseases, 2nd ed. Oxford: Blackwell, 1979:73-92.

2 Pittsley RA, Talal N. Neuromuscular complications of Sjögren's syndrome. In: Vinken PJ, Bruyn GW, eds. Handbook of Clinical Neurology. Vol. 39. Amsterdam: North Holland Publishing Co. 1980:419-33.

${ }^{3}$ Kaltreider HB, Talal N. The neuropathy of Sjögren's syndrome. Trigeminal nerve involvement. Ann Int Med 1969;70:751-62.

${ }^{4}$ Hull RG, Morgan SH, Harding AE, Hughes GRV.0 Sjögren's syndrome presenting as a severe sensor $\bar{P}$ neuropathy including involvement of the trigeminaf nerve. Br J Rheumatol 1984;23:301-3.

${ }^{s}$ Skoglund S. Anatomical and physiological studies of knee joint innervation in cat. Acta Physiol Scan 1956;36 (supp. 124): 1-101.

- Alexander GE, Provost TT, Stevens MB, Alexander EI Sjögren's syndrome: central nervous system manifes tations. Neurology (NY) 1981;31:1391-6.

7 Alexander EL, Provost TT, Stevens MB, Alexander GE. Neurological complications of primary Sjögren's syndrome. Medicine (Baltimore) 1982;61:247-57.

${ }^{8}$ Pallis CA, Scott JT. Peripheral neuropathy in rheumatoid arthritis. $\mathrm{Br}$ Med J 1965;i:1141-7.

${ }^{y}$ Weber FP. Sjögren's syndrome, especially its non-ocular features. Br J Ophthalmol 1945;29:299-312.

${ }^{10}$ Attwood W, Poser CM. Neurologic complications of $\overrightarrow{\vec{A}}$ Sjögren's syndrome. Neurology (Minneap) 1961; 11: 1034-41.

11 Thompson HS. Adie's syndrome: some new observations. Trans Am Ophth Soc 1977;75:587-626.

12 Conn DC, Dyck PJ. Angiopathic neuropathy in connective tissue diseases. In: Dyck PJ, Thomas PK, Lambert EH, Bunge R, eds. Peripheral Neuropathy, 2nd ed. Philadelphia: Saunders, 1984:2027-43. 